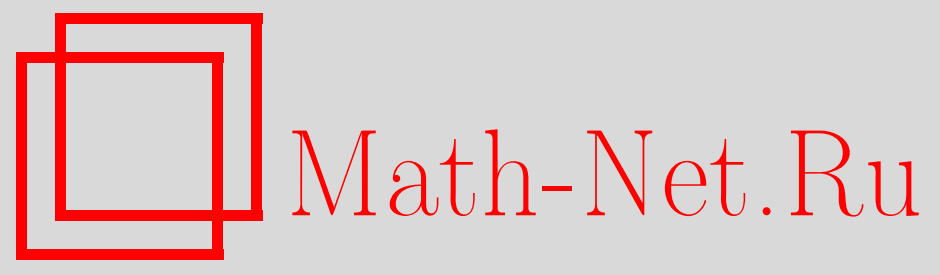

В. Г. Мосин, Примитивные идеалы в алгебре регулярных функций на квантовых $(m \times n)$-матрицах, Матем. заметкu, 2002, том 71, выпуск 2, 318-320

DOI: https://doi.org/10.4213/mzm644

Использование Общероссийского математического портала Math-Net.Ru подразумевает, что вы прочитали и согласны с пользовательским соглашением http://www . mathnet.ru/rus/agreement

Параметры загрузки:

IP : 52.6 .47 .48

26 апреля 2023 г., 14:10:32

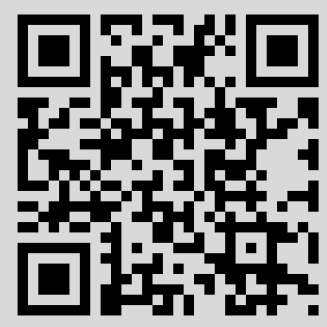




\section{ПРИМИТИВНЫЕ ИДЕАЛЫ В АЛГЕБРЕ РЕГУЛЯРНЫХ ФУНКЦИЙ НА КВАНТОВЫХ $(m \times n)$-МАТРИЦАХ}

\section{В.Г. Мосин}

1. Известно (см. [1]-[3]), что между примитивными идеалами в алгебре функций на квантовой группе и симплектическими листами в ассоциированной пуассоновой структуре имеется взаимно однозначное соответствие. В настоящей работе мы обобщаем этот результат на случай квантовых $(m \times n)$-матриц.

Пусть $\mathscr{V}$ - множество комплексных $(m \times n)$-матриц $(m \leqslant n)$, невырожденных в том смысле, что все их миноры максимального порядка отличны от нуля, $\mathbf{C}[\mathscr{V}]$ - алгебра регулярных функций на $\mathscr{V}$, $\mathbf{C}_{q}[\mathscr{V}]$ - ее квантовьй аналог. Мы разбиваем $\operatorname{Prim} \mathbf{C}_{q}[\mathscr{V}]$ на классы (предложение 4.1) подобно тому, как это сделано в [2], и доказьваем теорему 5.3 о взаимно однозначном соответствии между примитивными идеалами из $\mathbf{C}_{q}[\mathscr{V}]$ и симплектическими листами в $\mathscr{V}$. Кроме того, мы выводим формулу размерности симплектического листа (теорема 6.1 ), которая в частном случае $m=n$ дает известное (см. [1]-[3]) значение размерности листа на группе.

2. Обозначим через $\mathscr{M}$ алгебру регулярных функций на линейном пространстве $\mathscr{U}$ комплексных $(m \times n)$-матриц. Ее квантовьм аналогом служит алгебра $\mathscr{M}_{q}$ (см. [4, определение 1.2]), определенная как ассоциативная $\mathbf{C}$-алгебра, порожденная $m n$ образующими $X_{i}^{j}, 1 \leqslant i \leqslant m, 1 \leqslant j \leqslant n$, с квадратичными соотношениями

$$
\begin{aligned}
X_{i}^{j} X_{k}^{j} & =q^{-1} X_{k}^{j} X_{i}^{j} & & \forall i<k, \\
X_{i}^{j} X_{i}^{k} & =q^{-1} X_{i}^{k} X_{i}^{j} & & \forall j<k, \\
X_{i}^{j} X_{l}^{k} & =X_{l}^{k} X_{i}^{j} & & \forall i<l, j>k, \\
{\left[X_{i}^{j}, X_{l}^{k}\right] } & =\left(q^{-1}-q\right) X_{i}^{k} X_{l}^{j} & & \forall i<l, j<k,
\end{aligned}
$$

где $q$ - общий параметр квантования, т.е. переменная или комплексное число, не являющееся корнем из 1. Тогда алгебры $\mathbf{C}[\mathscr{V}]$ и $\mathbf{C}_{q}[\mathscr{V}]$ определяются как соответствующие локализации алгебр $\mathscr{M}$ и $\mathscr{M}_{q}$ по всем минорам (квантовьгм минорам в случае $\left.\mathbf{C}_{q}[\mathscr{V}]\right)$ порядка $m$.

3. Имея коммутативную алгебру $\mathbf{C}[\mathscr{V}]$ и ее деформацию $\mathbf{C}_{q}[\mathscr{V}]$, введем скобку Пуассона в $\mathbf{C}[\mathscr{V}]$ (как это делается, например, в $[5, \S 6]$ ) по формуле

$$
\forall f, g \in \mathbf{C}[\mathscr{V}] \quad\{f, g\}=\lim _{q \rightarrow 1} \frac{f * g-g * f}{q-1},
$$

где * означает умножение в $\mathbf{C}_{q}[\mathscr{V}]$. Тем самым, алгебра $\mathbf{C}[\mathscr{V}]$ становится пуассоновой алгеброй, ассоциированной с $\mathbf{C}_{q}[\mathscr{V}]$.

4. Будем обозначать $D_{I}^{J}$ (соответственно $C_{I}^{J}$ ) минор в $\mathscr{M}$ (соответственно квантовый минор в $\mathscr{M}_{q}$ ), построенный на строках с номерами из мультииндекса $I=\left\{i_{1}, \ldots, i_{k}\right\}$ и на столбцах с номерами из $J=\left\{j_{1}, \ldots, j_{k}\right\}$. Все мультииндексы предполагаются упорядоченньми, $E_{k}=\{1, \ldots, k\}$. Для подстановки $y$ обозначим $y(I)$ мультииндекс с компонентами $y\left(i_{1}\right), \ldots, y\left(i_{k}\right)$. Пару подстановок $w_{+}, w_{-}$из $S_{m}$ будем обозначать $w$. В алгебре $\mathscr{M}$ положим

(C) в. Г. Мосин

2002 


$$
\begin{array}{ll}
R_{w_{+}}^{+}=\bigcup_{i=1}^{m-1}\left\{D_{K}^{E_{i}} \mid K>w_{+}^{-1}\left(E_{i}\right)\right\}, & S_{w_{+}}^{+}=\bigcup_{i=1}^{m-1}\left\{D_{w_{+}^{-1}\left(E_{i}\right)}^{E_{i}}\right\}, \\
R_{w_{-}}^{-}=\bigcup_{i=1}^{m-1}\left\{D_{K}^{E_{n} \backslash E_{n-i}} \mid K<w_{-}^{-1}\left(E_{m} \backslash E_{m-i}\right)\right\}, & S_{w_{-}}^{-}=\bigcup_{i=1}^{m-1}\left\{D_{w_{-}^{-1}\left(E_{m} \backslash E_{m-i}\right)}^{E_{n} \backslash E_{n-i}}\right\},
\end{array}
$$

где $<$ (или $>$ ) означает лексикографический порядок, и пусть $\mathscr{V}_{w}$ - алгебраическое подмногообразие в $\mathscr{V}$, определенное условиями $D_{I}^{J}=0$ для всех $D_{I}^{J} \in R w, D_{I}^{J} \neq 0$ для всех $D_{I}^{J} \in S w$, где $R_{w}=R_{w_{+}}^{+} \cup R_{w_{-}}^{-}, S_{w}=S_{w_{+}}^{+} \cup S_{w_{-}}^{-}$. С другой стороны, в алгебре $\mathscr{M}_{q}$ обозначим через $P_{w}, T_{w}$ множества квантовых миноров, аналогичные соответственно множествам $R_{w}, S_{w}$ в $\mathscr{M}$. Пусть $\mathscr{P}_{w}$ - левый идеал в $\mathscr{M}_{q}$, порожденный $P_{w} ; \mathscr{T}_{w}$-мультипликативное подмножество в $\mathscr{M}_{q}$, порожденное $T_{w}$. Заметим, что $\mathscr{P}_{w}$, определенньй как левый идеал в $\mathscr{M}_{q}$, на самом деле является двусторонним. Положим

$$
\begin{aligned}
\operatorname{Symp}_{w} \mathscr{V} & =\left\{\mathscr{L} \in \operatorname{Symp} \mathscr{V} \mid \mathscr{L} \subseteq \mathscr{V}_{w}\right\} \\
\operatorname{Prim}_{w} \mathbf{C}_{q}[\mathscr{V}] & =\left\{\mathscr{P} \in \operatorname{Prim} \mathbf{C}_{q}[\mathscr{V}] \mid \mathscr{P} \supseteq \mathscr{P}_{w}, \mathscr{P} \cap \mathscr{T}_{w}=\varnothing\right\}
\end{aligned}
$$

и пусть $\sqcup$ означает объединение попарно не пересекающихся множеств.

ПРЕДЛОЖЕНИЕ 4.1. Имеют место соотношения:

1) $\operatorname{Symp} \mathscr{V}=\bigsqcup_{w \in S_{m}} \times S_{m} \operatorname{Symp}_{w} \mathscr{V}$;

2) $\operatorname{Prim} \mathbf{C}_{q}[\mathscr{V}]=\bigsqcup_{w \in S_{m} \times S_{m}} \operatorname{Prim}_{w} \mathbf{C}_{q}[\mathscr{V}]$

5. Так как число классов конечно, то имеется биекция между классами и важно установить биекцию между примитивными идеалами и симплектическими листами внутри данного класса. Для этого построим вспомогательню алгебру $\mathscr{A}_{w}$ и соответствующее ей вспомогательное геометрическое множество $\mathscr{U}_{w}$.

Пусть $S_{0}=\left\{D_{E_{m}}^{E_{m}}, D_{E_{m}}^{\{2,3, \ldots, m-1\}}, \ldots, D_{E_{m}}^{E_{n} \backslash E_{n-m}}\right\}$. Обозначим через $\bar{S}_{w}$ множество всех главных миноров всех миноров из $S_{0} \cup S_{w}$. Тогда $\mathcal{U}_{w}$ - это алгебраическое подмногообразие в $\mathcal{U}$ (но не в $\mathscr{V}$ ), определенное условиями $D_{I}^{J}=0$ для всех $D_{I}^{J} \in R_{w}, D_{I}^{J} \neq 0$ для всех $D_{I}^{J} \in \bar{S}_{w}$. С

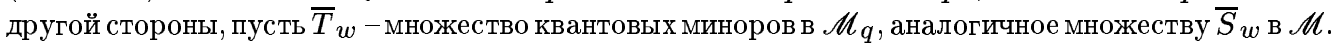
Тогда $\mathscr{A}_{w}=\left(\mathscr{M}_{q} / \mathscr{P}_{w}\right)_{\overline{\mathscr{T}}_{w}}$, где $\overline{\mathscr{T}}_{w}$-мульипликативное подмножество в $\mathscr{M}_{q}$, порожденное $\bar{T} w$.

Лемма 5.1. Имеют место соотношения:

1) $\operatorname{Symp}_{w} \mathscr{V} \simeq \operatorname{Symp} \mathscr{U}_{w}$

2) $\operatorname{Prim}_{w} \mathbf{C}_{q}[\mathscr{V}] \simeq \operatorname{Prim} \mathscr{A}_{w}$.

ПредЛОЖенИЕ 5.2. 1) Алгебра $\mathbf{C}_{q}\left[\mathscr{U}_{w}\right]$ регулярных функиий на $\mathscr{U}_{w}$ является лорановской алгеброй, порожденной минорами из $\bar{S}_{w}$.

2) Алгебра $\mathscr{A}_{w}$ является скрученной лорановской алгеброй, порожденной квантовыми минорами из $\bar{T}_{w}$.

Tеорема 5.3. Имеется биекиия $\beta$ межлду $\operatorname{Prim} \mathbf{C}_{q}[\mathscr{V}]$ u Symp $\mathscr{V}$ maкая, ито

1) $\beta\left(\operatorname{Prim}_{w} \mathbf{C}_{q}[\mathscr{V}]\right)=\operatorname{Symp}_{w} \mathscr{V}$;

2) $\operatorname{dim} \beta(\mathscr{P})=\mathrm{GKdim} \mathbf{C}_{q}[\mathscr{V}] / \mathscr{P}$ для всех $\mathscr{P} \in \operatorname{Prim} \mathbf{C}_{q}[\mathscr{V}]$, əде GKdim означает размерность Гельфанда-Кириллова.

Идея доказательства состоит в том, что, с одной стороны, алгебра $\mathscr{A} w$ является скрученной и, следовательно, все ее примитивные идеалы порождаются максимальньми идеалами ее центра (см. $[6,2.3])$. С другой стороны, симплектические листы в $\mathscr{U}_{w}$ определяются набором функций Казимира (см. $[5, \S 2])$, играющих в пуассоновой алгебре $\mathbf{C}_{q}\left[\mathscr{U}_{w}\right]$ роль центральных элементов. Если пара образующих $C_{I}^{J}, C_{K}^{L}$ алгебры $\mathscr{A}_{w}$ связана соотношением

$$
C_{I}^{J} C_{K}^{L}=q^{\varphi_{I K}^{J}} C_{K}^{L} C_{I}^{J},
$$

то предельный переход, данньй в п. 3 , показьвает, что для соответствующих образующих $D_{I}^{J}, D_{K}^{L}$ алгебры $\mathbf{C}\left[\mathcal{U}_{w}\right]$ справедливо соотношение

$$
\left\{D_{I}^{J}, D_{K}^{L}\right\}=\varphi_{I K}^{J L} D_{I}^{J} D_{K}^{L} .
$$


Поэтому как образующие Center $\mathscr{A}_{w}$, так и образующие Cazimir $\mathbf{C}_{q}\left[\mathscr{U}_{w}\right]$ определяются целыми решениями одной и той же системы $\Phi \bar{t}=0$, откуда следует биекция. Утверждение о размерности вытекает из того, что GKdim Center $\mathscr{A}_{w}=\operatorname{dim} \operatorname{ker} \Phi=\operatorname{GKdim} \operatorname{Cazimir} \mathbf{C}_{q}\left[\mathscr{U}_{w}\right]$.

6. Пусть $p$ - частное, а $r$ - остаток от деления $n$ на $m$. Обозначим

$$
w_{r}=\left(\begin{array}{cc}
0 & e_{m-r} \\
e_{r} & 0
\end{array}\right), \quad \varepsilon_{r}=\left(\begin{array}{cc}
-e_{r} & 0 \\
0 & e_{m-r}
\end{array}\right),
$$

где $e_{j}$ - единичная матрица порядка $j$.

Теорема 6.1. Если $\mathscr{L}_{w}-$ симплектический лист из $\operatorname{Symp}_{w} \mathscr{V}$, mo

$$
\operatorname{dim} \mathscr{L}_{w}=m(n-m)+l(w)+\operatorname{rk}\left(w_{+} w_{-}^{-1} w_{r}+(-1)^{p} \varepsilon_{r}\right),
$$

где $l(w)$ означает сумму чисел инверсий в подстановках $w_{+}, w_{-}$.

В [1]-[3] изучались примитивные идеалы на квантовых группах и их связь с симплектическими листами. В частности, на группе симплектический лист типа $w$ имеет размерность $l(w)+$ $\operatorname{rk}\left(w_{+} w_{-}^{-1}-\mathrm{Id}\right)$ (см., например, [1, теорема A.3.2]). Полагая в теореме $6.1 m=n$, нетрудно убедиться в согласованности наших результатов с результатами [1]-[3].

Автор выражает искреннюю признательность своему научному руководителю А. Н. Панову за внимание и поддержку, а также Т. Левассюру и А. Жозефу за то, что они прислали ему оттиски своих работ.

\section{СПИСОК ЦИТИРОВАННОЙ ЛИТЕРАТУРЫ}

1. Hodges T. J., Levasseur T. // Comm. Math. Phys. 1993. V. 156. P. 581-605. 2. Hodges T.J., Levasseur T. // J. Algebra. 1994. V. 168. P. 455-468. 3. Joseph A. // J. Algebra. 1994. V. 169. Р. 441-511. 4. Мосин В. Г., Панов А. Н. // Матем. сб. 1996. Т. 187. №6. C. 53-72. 5. Vaisman I. Lectures on the Geometry of Poisson Manifolds. Progress in Math. V. 118. Basel-Boston-Berlin: Birkhäuser Verlag, 1994. 